\title{
EXOTIME: searching for planets around pulsating subdwarf B stars
}

\author{
Sonja Schuh • Roberto Silvotti • Ronny Lutz • Björn Loeptien • Elizabeth M. Green • \\ Roy H. Østensen • Silvio Leccia - Seung-Lee Kim • Gilles Fontaine • \\ Stéphane Charpinet • Myriam Francœur • Suzanna Randall • \\ Cristina Rodríguez-López • Valerie van Grootel • Andrew P. Odell • Margit Paparó • \\ Zsófia Bognár · Péter Pápics • Thorsten Nagel • Benjamin Beeck • \\ Markus Hundertmark • Thorsten Stahn • Stefan Dreizler • Frederic V. Hessman • \\ Massimo Dall'Ora • Dario Mancini · Fausto Cortecchia • Serena Benatti • \\ Riccardo Claudi · Rimvydas Janulis \\ Received: 24 December 2009 / Accepted: 3 March 2010 / Published online: 27 March 2010 \\ (C) The Author(s) 2010. This article is published with open access at Springerlink.com
}

\author{
S. Schuh $\cdot$ T. Nagel \\ Institut für Astronomie und Astrophysik, Kepler Center for Astro \\ and Particle Physics, Eberhard-Karls-Universität, Sand 1, 72076 \\ Tubingen, Germany \\ S. Schuh $(\bowtie) \cdot$ R. Lutz $\cdot$ B. Loeptien $\cdot$ B. Beeck · \\ M. Hundertmark · S. Dreizler · F.V. Hessman \\ Institut für Astrophysik, Georg-August-Universität Göttingen, \\ Friedrich-Hund-Platz 1, 37077 Goettingen, Germany \\ e-mail: schuh@astro.physik.uni-goettingen.de \\ R. Silvotti \\ Istituto Nazionale di Astrofisica (INAF), Osservatorio \\ Astronomico di Torino, Strada Osservatorio 20, 10025, Pino \\ Torinese, Italy \\ R. Silvotti - S. Leccia · M. Dall'Ora · D. Mancini · F. Cortecchia \\ Istituto Nazionale di Astrofisica (INAF), Osservatorio \\ Astronomico di Capodimonte, via Moiariello 16, 80131 Napoli, \\ Italy \\ R. Lutz · T. Stahn \\ Max-Planck-Institut für Sonnensystemforschung, \\ Max-Planck-Strafle 2, 37191 Katlenburg-Lindau, Germany \\ E.M. Green \\ Steward Observatory, University of Arizona, 933 North Cherry \\ Avenue, Tucson, AZ 85721, USA \\ R.H. Østensen · P. Pápics \\ Instituut voor Sterrenkunde, K.U. Leuven, Celestijnenlaan 200D, \\ 3001 Leuven, Belgium

\section{S.-L. Kim} \\ Korea Astronomy and Space Science Institute, Daejeon 305-348, \\ Korea \\ G. Fontaine $\cdot$ M. Francœur \\ Département de Physique, Université de Montréal, C.P. 6128 \\ Succ. Centre-Ville, Montréal H3C 3J7, Canada \\ S. Charpinet · C. Rodríguez-López · V. van Grootel \\ Laboratoire d'Astrophysique de Toulouse-Tarbes, Université de \\ Toulouse, CNRS, 14 Avenue Édouard Belin, 31400 Toulouse, \\ France \\ S. Randall \\ ESO, Karl-Schwarzschild-Str. 2, 85748 Garching near Munich, \\ Germany \\ C. Rodríguez-López \\ Departamento de Física Aplicada, Universidade de Vigo, Vigo \\ 36310, Spain \\ A.P. Odell \\ Northern Arizona University, Flagstaff, AZ 86011, USA \\ M. Paparó · Z. Bognár \\ Konkoly Observatory of the Hungarian Academy of Sciences, \\ P.O. Box 67, 1525 Budapest XII, Hungary \\ P. Pápics \\ Eötvös Loránd University Faculty of Science, Pázmány Péter \\ sétány 1/A, 1117 Budapest, Hungary \\ S. Benatti \\ CISAS, Università degli studi di Padova, via Venezia 15, 35131 \\ Padova, Italy \\ R. Claudi \\ Istituto Nazionale di Astrofisica (INAF), Osservatorio \\ Astronomico di Padova, vicolo Osservatorio 5, 35122 Padova, \\ Italy \\ R. Janulis \\ Institute of Theoretical Physics and Astronomy, Vilnius \\ University, 12 A. Gostauto Street, 01108 Vilnius, Lithuania
}


Abstract In 2007, a companion with planetary mass was found around the pulsating subdwarf B star V391 Pegasi with the timing method, indicating that a previously undiscovered population of substellar companions to apparently single subdwarf B stars might exist. Following this serendipitous discovery, the EXOTIME (http://www.na.astro.it/ silvotti/exotime/) monitoring program has been set up to follow the pulsations of a number of selected rapidly pulsating subdwarf B stars on time scales of several years with two immediate observational goals:

(1) determine $\dot{P}$ of the pulsational periods $P$

(2) search for signatures of substellar companions in $\mathrm{O}-$ $\mathrm{C}$ residuals due to periodic light travel time variations, which would be tracking the central star's companioninduced wobble around the centre of mass

These sets of data should therefore, at the same time, on the one hand be useful to provide extra constraints for classical asteroseismological exercises from the $\dot{P}$ (comparison with "local" evolutionary models), and on the other hand allow one to investigate the preceding evolution of a target in terms of possible "binary" evolution by extending the otherwise unsuccessful search for companions to potentially very low masses.

While timing pulsations may be an observationally expensive method to search for companions, it samples a different range of orbital parameters, inaccessible through orbital photometric effects or the radial velocity method: the latter favours massive close-in companions, whereas the timing method becomes increasingly more sensitive toward wider separations.

In this paper we report on the status of the on-going observations and coherence analysis for two of the currently five targets, revealing very well-behaved pulsational characteristics in HS 0444+0458, while showing HS 0702+6043 to be more complex than previously thought.

Keywords Stars: subdwarfs - Stars: oscillations - Stars: evolution - Stars: planetary systems · Individual: HS 2201+2610 - Individual: HS 0702+6043 - Individual: HS $0444+0458$

\section{Introduction}

While $\dot{P}$ measurements exist for a number of pulsating white dwarfs (e.g. Kepler et al. 1991, 2000, 2005; Costa et al. 1999; Costa and Kepler 2008; Mullally et al. 2008), similar measurements have only been published for one pulsating subdwarf B star so far, V391 Pegasi (hereafter HS 2201+2610).

HS $2201+2610$ was first discovered to be a rapidly pulsating subdwarf B star by Østensen et al. (2001a). Silvotti et al. $(2002,2007)$ were able to derive $\dot{P}$ values for the two strongest pulsation modes, and found an additional pattern in the $\mathrm{O}-\mathrm{C}$ diagrams that revealed the presence of a giant planet in a 3.2 year orbit. Additional slow pulsations were subsequently reported by Lutz et al. (2009b).

From theoretical considerations, both the rapid p-mode as well as the slow g-mode oscillations should be very stable in the pulsating subdwarf B stars (stable in phase, i.e. coherent over time scales of many years, as well as stable in amplitude) due to the identical underlying driving: a $\kappa$ mechanism acting on the $Z$ bump. Time dependency of phases and amplitudes can, however, be introduced through nonlinear effects; the resulting mode coupling then starts to invalidate the initial simple assumption. It is therefore crucial to identify target stars that show a stable behaviour.

Under the right conditions, this provides the possibility to obtain meaningful results from long-term photometric monitoring of rapidly pulsating sdB stars: it allows to search for slow changes in pulsation periods due to evolutionary effects, testing the time scales in sdB evolutionary models, and may be used as an additional constraint for sdB asteroseismological models. It also allows to use the star as a clock that provides a regular timing signal, which can be exploited to search for small periodic deviations from the mean pulsation maxima arrival times. Such periodic deviations can be caused by a varying light travel time and may hence indicate a wobble of the pulsating subdwarf B star's location due to one or more low-mass companions, as is the case for HS 2201+2610. This timing method is sensitive to planetary masses and large period ranges not easily accessible with other methods.

Given the observational efforts required, the detection rate of low-mass companions in wide orbits is surprisingly high so far. Besides timing of pulsations (Silvotti et al. 2007), timing of the sharp eclipses in HW Vir type systems has been used to detect tertiary bodies (Lee et al. 2009; Qian et al. 2009). Furthermore, Geier et al. (2009) have even successfully employed the radial velocity method to detect a close substellar companion to HD 149382.

As it has been suggested that the influence of substellar companions or large planetary companions may be decisive for the evolution of subdwarf B stars (see Discussion in Sect. 6), the EXOTIME program has been set up to find more such objects. We discuss the target selection in Sect. 2. However, as amplitude variations have repeatedly been reported in sdB pulsators (e.g. Kilkenny 2010), it is crucial to carefully check the stability of the targets first of all. This contribution mainly discusses the current status of these investigations for two of our objects, HS 0444+0458 and HS 0702+6043 (Sect. 3).

HS 0444+0458 was first discovered to pulsate by Østensen et al. (2001b), and has been further characterised by Reed et al. (2007). Rapid oscillations were discovered in 
Table 1 Overview of EXOTIME targets

\begin{tabular}{llllll}
\hline Target & Coordinates & (Equinox 2000.) & $m_{\mathrm{B}}$ & Status & \\
\hline HS 2201+2610 & $22: 04: 12.0$ & $+26: 25: 07$ & 14.3 & collecting data & planet candidate published, sin $i$ unknown \\
HS 0702+6043 & $07: 07: 09.8$ & $+60: 38: 50$ & 14.7 & collecting data & see Sects. 3.1 and 4 \\
HS 0444+0458 & $04: 47: 18.6$ & $+05: 03: 35$ & 15.2 & collecting data & see Sect. 3.2 \\
EC 09582-1137 & $10: 00: 41.8$ & $-11: 51: 35$ & 15.0 & collecting data & \\
PG 1325+101 & $13: 27: 48.6$ & $+09: 54: 52$ & 13.8 & collecting data & \\
\hline
\end{tabular}

HS 0702+6043 by Dreizler et al. (2002), simultaneous slow oscillations were reported by Schuh et al. (2006). The ongoing EXOTIME observations for HS 0702+6043 have also previously been summarised by Lutz et al. (2008b, 2009a).

\section{Observational data}

\subsection{Target selection}

Similar to the criteria applied when first selecting HS 2201+2610 for long-term monitoring, we considered two sets of characteristics to choose further targets. The first set concerns the pulsations: frequencies, amplitudes and phases are obviously required to be stable over long periods of time. Although there are early indicators if these criteria are not fulfilled, it should be clear these properties can only be truly verified, in particular for the phases, from long-term monitoring data in hindsight (see Sect. 3).

Using target properties partly taken from Reed et al. (2007), we selected candidates with few but preferably more than one or two frequencies, in order to be able to fully resolve the frequency spectrum during short individual runs while also retaining the possibility to obtain an independent $\mathrm{O}-\mathrm{C}$ measurement from each individual frequency. For the amplitudes we leaned toward medium values, avoiding, where possible, very small amplitudes which would have $\mathrm{S} / \mathrm{N}$ issues, as well as very large ones where one might have to start worrying about non-linearity effects.

We also used Reed et al.'s listing to consider the amplitude stability criterion given by Christensen-Dalsgaard et al. (2001), which for oscillations similar to solar-like ones identifies the stochastically excited pulsations. While this quantitative distinction for the underlying type of driving has, not surprisingly at all, been previously shown inadequate for the opacity-driven subdwarf B pulsators, we still found it helpful to restrict our list of objects to those with $\sigma(A) /\langle A\rangle<0.5$. We took this as a purely observational value that overall should favour the more stable pulsators.

The second set of selection criteria was concerned with more practical questions, looking for the brighter ones of the available candidates and including preferentially northern hemisphere targets. Compromising between all of these considerations, we selected the objects in Table 1 as the initial list of targets to be observed.

\subsection{Observations}

Due to not only the different magnitudes and pulsation amplitudes as well as the spatial distribution of the targets, but also due to the long-term nature of the project and the changing availability of telescopes, the observations in the data bases for our target objects come from a variety of sites, instruments, and observers. The bulk of observations for HS 2201+2610 have been published with extensive observing logs by Silvotti et al. (2002, 2007). Further data is still being collected and analysed in order to confirm the future evolution in the $\mathrm{O}-\mathrm{C}$ diagram as predicted from the known orbital motion, as well as to search for possible further companions at different orbital periods.

The two other targets with the best coverage so far (after HS 2201+2610) are HS 0702+6043 and HS 0444+0458. For these two targets, the full observing logs up to date are provided in Table 2 (HS 0444+0458) and Table 3 (HS 0702+6043), respectively. The observations available for these two targets come from telescopes ranging from $0.5 \mathrm{~m}$ to $3.6 \mathrm{~m}$ in aperture, and were all obtained with CCD detectors, almost all of the time through B filters, and with exposure times between $5 \mathrm{~s}$ and $40 \mathrm{~s}$.

We note that the observations for HS $0702+6043$ comprise a very large and very high-quality data set obtained by Green et al. in 2007/2008 ("Mt. Bigelow data set") primarily for asteroseismological purposes, which is presented in detail by Francœur et al. (2010) elsewhere in this issue. We include and examine it here exclusively under the aspect of long-term changes.

\subsection{Data reduction}

Light curves were extracted from (bias-, dark- and flat-fieldcorrected) CCD frames with aperture photometry packages, usually directly by the observers. Relative light curves 
Table 2 Photometric data archive for HS0444+0458

\begin{tabular}{|c|c|c|c|c|c|c|c|c|c|c|}
\hline Year & Month & Day & $L[\mathrm{~h}]$ & $N$ & $\sigma_{\text {res }}$ & $\operatorname{Exp}[\mathrm{s}]$ & Site $^{\mathrm{a}}$ & Inst. & Filter & Observer $^{\mathrm{b}}$ \\
\hline \multirow[t]{11}{*}{2008} & \multirow[t]{6}{*}{ Aug } & 19 & 0.5 & 107 & 0.0069 & 5 & TNG $3.6 \mathrm{~m}$ & DOLORES & $\mathrm{B}$ & $\mathrm{RS}, \mathrm{SL}$ \\
\hline & & 21 & 1.0 & 237 & 0.0076 & 5 & TNG $3.6 \mathrm{~m}$ & DOLORES & B & $\mathrm{RS}, \mathrm{SL}$ \\
\hline & & 24 & 0.7 & 173 & 0.0060 & 5 & TNG $3.6 \mathrm{~m}$ & DOLORES & B & RS, SL \\
\hline & & 26 & 1.0 & 224 & 0.0051 & 5 & TNG $3.6 \mathrm{~m}$ & DOLORES & B & RS, SL \\
\hline & & 27 & 2.1 & 357 & 0.0040 & 10 & TNG $3.6 \mathrm{~m}$ & DOLORES & $\mathrm{B}$ & $\mathrm{RS}, \mathrm{SL}$ \\
\hline & & 28 & 1.7 & 230 & 0.0042 & 10 & TNG $3.6 \mathrm{~m}$ & DOLORES & B & RS, SL \\
\hline & Sep & 29 & 2.2 & 171 & 0.0089 & 10 & $\mathrm{CA} 2.2 \mathrm{~m}$ & CAFOS & B & service $(\mathrm{FH})$ \\
\hline & \multirow[t]{3}{*}{ Oct } & 24 & 2.8 & 300 & 0.0191 & 12 & LOAO $1.0 \mathrm{~m}$ & $2 \mathrm{k} C \mathrm{CD}$ & B & SLK \\
\hline & & 26 & 4.9 & 588 & 0.0232 & 12 & LOAO $1.0 \mathrm{~m}$ & $2 \mathrm{k} C \mathrm{CD}$ & B & SLK \\
\hline & & 29 & 1.2 & 163 & 0.0175 & 12 & LOAO $1.0 \mathrm{~m}$ & $2 \mathrm{k} C C D$ & $\mathrm{~B}$ & SLK \\
\hline & Nov & 21 & 3.2 & 263 & 0.0092 & 10 & CA $2.2 \mathrm{~m}$ & CAFOS & B & service (MA) \\
\hline \multirow[t]{12}{*}{2009} & \multirow[t]{2}{*}{ Jan } & 29 & 2.6 & 275 & 0.0239 & 20 & LOAO $1.0 \mathrm{~m}$ & $2 \mathrm{k} C C D$ & $\mathrm{~B}$ & SLK \\
\hline & & 31 & 4.7 & 497 & 0.0275 & 20 & LOAO $1.0 \mathrm{~m}$ & 2k CCD & B & SLK \\
\hline & \multirow[t]{3}{*}{ Mar } & 20 & 1.0 & 94 & 0.0090 & 10 & CA $2.2 \mathrm{~m}$ & CAFOS & B & service (MA) \\
\hline & & 21 & 1.3 & 135 & 0.0121 & 10 & CA $2.2 \mathrm{~m}$ & CAFOS & B & service (MA) \\
\hline & & 22 & 1.2 & 124 & 0.0099 & 10 & CA $2.2 \mathrm{~m}$ & CAFOS & B & service (MA) \\
\hline & \multirow[t]{7}{*}{ Oct } & 16 & 1.3 & 195 & 0.0138 & 20 & $\mathrm{M} / \mathrm{N} 1.2 \mathrm{~m}$ & $1 \mathrm{k} C C D$ & B & $\mathrm{RL}$ \\
\hline & & 18 & 1.6 & 242 & 0.0148 & 20 & $\mathrm{M} / \mathrm{N} 1.2 \mathrm{~m}$ & $1 \mathrm{k} C C D$ & B & RL \\
\hline & & 22 & 2.1 & 235 & 0.0102 & 10 & CA $2.2 \mathrm{~m}$ & CAFOS & B & service (AA) \\
\hline & & 23 & 2.9 & 330 & 0.0082 & 10 & CA $2.2 \mathrm{~m}$ & CAFOS & B & service (MA) \\
\hline & & 24 & 2.2 & 244 & 0.0075 & 10 & CA $2.2 \mathrm{~m}$ & CAFOS & B & service (MA) \\
\hline & & 25 & 1.7 & 104 & 0.0061 & 10 & CA $2.2 \mathrm{~m}$ & CAFOS & B & service (MA) \\
\hline & & 26 & 3.4 & 387 & 0.0086 & 10 & $\mathrm{CA} 2.2 \mathrm{~m}$ & CAFOS & $\mathrm{B}$ & service (MA) \\
\hline
\end{tabular}

${ }^{a}$ CA: Calar Alto Observatory, LOAO: Mt. Lemmon Optical Astronomy Observatory, M/N: MONET/North Telescope, TNG: Telescopio Nazionale Galileo

${ }^{\mathrm{b}}$ FH: Felipe Hoyo, MA: Manuel Alises, RL: Ronny Lutz, RS: Roberto Silvotti, SLK: Seung-Lee Kim, SL: Silvio Leccia

Table 3 Photometric data archive for HS 0702+6043, part a

\begin{tabular}{|c|c|c|c|c|c|c|c|c|c|c|}
\hline Year & Month & Day & $L[\mathrm{~h}]$ & $N$ & $\sigma_{\text {res }}$ & $\operatorname{Exp}[s]$ & Site $^{\mathrm{a}}$ & Inst. & Filter & Observer $^{\mathrm{b}}$ \\
\hline \multirow[t]{3}{*}{1999} & Dec & 06 & 5.0 & 943 & 0.0103 & 10 & $\mathrm{CA} 1.2 \mathrm{~m}$ & $\mathrm{CCD}$ & - & $\mathrm{SD}, \mathrm{SS}$ \\
\hline & & 07 & 1.9 & 414 & 0.0141 & 8 & CA $1.2 \mathrm{~m}$ & $\mathrm{CCD}$ & - & $\mathrm{SD}, \mathrm{SS}$ \\
\hline & & 08 & 1.4 & 241 & 0.0085 & 10 & CA $1.2 \mathrm{~m}$ & $\mathrm{CCD}$ & - & $\mathrm{SD}, \mathrm{SS}$ \\
\hline 2000 & Oct & 08 & 0.7 & 248 & 0.0027 & 10 & NOT $2.56 \mathrm{~m}$ & ALFOSC & $3 \mathrm{~W}$ & $\mathrm{R} \varnothing$ \\
\hline \multirow[t]{3}{*}{2004} & Feb & 04 & 7.3 & 685 & 0.0233 & 30 & Tue $0.8 \mathrm{~m}$ & ST7 & $\mathrm{G}$ & $\mathrm{TN}$ \\
\hline & & 09 & 5.8 & 244 & 0.0016 & 60 & $\mathrm{StB} 2.2 \mathrm{~m}$ & $2 \mathrm{k} C \mathrm{CD}$ & F555W & EMG \\
\hline & & 10 & 6.2 & 264 & 0.0014 & 60 & $\mathrm{StB} 2.2 \mathrm{~m}$ & 2k CCD & F555W & EMG \\
\hline 2005 & Jan & $17-27$ & 56.0 & 5981 & 0.0082 & 20,30 & CA $2.2 \mathrm{~m}$ & CAFOS & $\mathrm{B}$ & SS, TS \\
\hline \multirow[t]{5}{*}{2007} & Dec & 18 & 5.9 & 498 & 0.0442 & 40 & Tue $0.8 \mathrm{~m}$ & SBIG ST-L & B & $\mathrm{TN}$ \\
\hline & & 19 & 6.7 & 557 & 0.0589 & 40 & Tue $0.8 \mathrm{~m}$ & SBIG ST-L & $\mathrm{B}$ & $\mathrm{TN}$ \\
\hline & & 20 & 10.0 & 842 & 0.0994 & 40 & Tue $0.8 \mathrm{~m}$ & SBIG ST-L & $\mathrm{B}$ & $\mathrm{TN}$ \\
\hline & & 21 & 9.3 & 760 & 0.1350 & 40 & Tue $0.8 \mathrm{~m}$ & SBIG ST-L & B & $\mathrm{TN}$ \\
\hline & & & 12.1 & 621 & 0.0200 & & Goe $0.5 \mathrm{~m}$ & SBIG ST-L & B & SS, RL, RK \\
\hline
\end{tabular}


Table 3 (Continued)

\begin{tabular}{|c|c|c|c|c|c|c|c|c|c|c|}
\hline Year & Month & Day & $L[\mathrm{~h}]$ & $N$ & $\sigma_{\text {res }}$ & $\operatorname{Exp}[\mathrm{s}]$ & Site $^{\mathrm{a}}$ & Inst. & Filter & Observer $^{\mathrm{b}}$ \\
\hline 2007 & Nov & & & & & & & & & \\
\hline$\vdots$ & $\vdots$ & & 424.0 & 31638 & 0.0027 & 50 & $\mathrm{MtB} 1.55 \mathrm{~m}$ & MONT 4k & S 8612 & EMG \& coll. \\
\hline 2008 & Mar & & & & & & & & & \\
\hline \multirow[t]{32}{*}{2008} & Feb & 08 & 7.2 & 601 & 0.0124 & 40 & Tue $0.8 \mathrm{~m}$ & SBIG ST-L & B & $\mathrm{TN}$ \\
\hline & & 09 & 7.1 & 590 & 0.0121 & 40 & Tue $0.8 \mathrm{~m}$ & SBIG ST-L & B & $\mathrm{TN}$ \\
\hline & & & 8.9 & 642 & 0.0381 & 35 & Goe $0.5 \mathrm{~m}$ & SBIG ST-L & B & RL, BB \\
\hline & & 10 & 5.8 & 488 & 0.0134 & 40 & Tue $0.8 \mathrm{~m}$ & SBIG ST-L & B & $\mathrm{TN}$ \\
\hline & & & 12.2 & 910 & 0.0324 & 35 & Goe $0.5 \mathrm{~m}$ & SBIG ST-L & B & $\mathrm{RL}, \mathrm{MH}$ \\
\hline & & 11 & 4.5 & 338 & 0.0439 & 35 & Goe $0.5 \mathrm{~m}$ & SBIG ST-L & $\mathrm{B}$ & $\mathrm{RL}, \mathrm{MH}$ \\
\hline & & 13 & 1.1 & 86 & 0.0749 & 35 & Goe $0.5 \mathrm{~m}$ & SBIG ST-L & B & RL \\
\hline & & 15 & 5.4 & 379 & 0.0552 & 35 & Goe $0.5 \mathrm{~m}$ & SBIG ST-L & B & RL, SW \\
\hline & & 29 & 3.7 & 360 & & 20 & Loi $1.5 \mathrm{~m}$ & & B & service (ADB) \\
\hline & Mar & 01 & 5.7 & 451 & & 25 & Loi $1.5 \mathrm{~m}$ & & B & service (ADB) \\
\hline & & 03 & 5.1 & 429 & & 25 & Loi $1.5 \mathrm{~m}$ & & B & service (ADB) \\
\hline & & 05 & 0.6 & 47 & 0.0238 & 35 & Goe $0.5 \mathrm{~m}$ & SBIG ST-L & B & RL, SS \\
\hline & & 13 & 3.7 & 126 & 0.0069 & 30 & Asi 1.8 & Afosc & $\mathrm{R}$ & $\mathrm{SB}, \mathrm{RC}$ \\
\hline & & & 2.3 & 248 & 0.0105 & 30 & Kon $1.0 \mathrm{~m}$ & PI VA 1300B & B & MP, PP \\
\hline & & 15 & 4.6 & 597 & 0.0095 & 25 & Kon $1.0 \mathrm{~m}$ & PI VA 1300B & B & MP, PP \\
\hline & & 18 & 3.7 & 348 & 0.0111 & 35 & Kon $1.0 \mathrm{~m}$ & PI VA 1300B & B & MP, PP \\
\hline & & 19 & 1.1 & 137 & 0.0088 & 25 & Kon $1.0 \mathrm{~m}$ & PI VA 1300B & B & MP, PP \\
\hline & May & 11 & 3.0 & 204 & 0.0315 & 40 & Goe $0.5 \mathrm{~m}$ & SBIG ST-L & B & SW \\
\hline & & 12 & 1.2 & 160 & 0.0380 & 40 & Goe $0.5 \mathrm{~m}$ & SBIG ST-L & B & SW \\
\hline & & 13 & 3.2 & 266 & 0.0254 & 40 & Tue $0.8 \mathrm{~m}$ & SBIG ST-L & B & $\mathrm{AH}$ \\
\hline & Oct & 15 & 1.3 & 113 & 0.0085 & 10 & CA $2.2 \mathrm{~m}$ & CAFOS & B & service (LM) \\
\hline & & 16 & 0.8 & 65 & 0.0101 & 10 & CA $2.2 \mathrm{~m}$ & CAFOS & B & service (MA) \\
\hline & & 17 & 0.5 & 48 & 0.0152 & 10 & CA $2.2 \mathrm{~m}$ & CAFOS & B & service (MA) \\
\hline & & 18 & 1.6 & 143 & 0.0068 & 10 & CA $2.2 \mathrm{~m}$ & CAFOS & B & service (MA) \\
\hline & & 25 & 2.6 & 285 & 0.0089 & 30 & Kon $1.0 \mathrm{~m}$ & PI VA 1300B & B & $\mathrm{ZB}$ \\
\hline & & & 4.4 & 518 & 0.0107 & 15 & LOAO $1.0 \mathrm{~m}$ & $2 \mathrm{k} C \mathrm{CD}$ & B & SLK \\
\hline & & 28 & 2.9 & 326 & 0.0104 & 15 & LOAO $1.0 \mathrm{~m}$ & $2 \mathrm{k} C \mathrm{CD}$ & $\mathrm{B}$ & SLK \\
\hline & & 30 & 3.9 & 455 & 0.0104 & 15 & LOAO $1.0 \mathrm{~m}$ & 2k CCD & B & SLK \\
\hline & Nov & 21 & 3.0 & 254 & 0.0048 & 10 & CA $2.2 \mathrm{~m}$ & CAFOS & B & service (MA) \\
\hline & & 23 & 5.7 & 496 & 0.0064 & 10 & CA $2.2 \mathrm{~m}$ & CAFOS & B & service (MA) \\
\hline & Dec & 16 & 3.0 & 243 & 0.0068 & 25 & $\mathrm{M} / \mathrm{N} 1.2 \mathrm{~m}$ & 1k CCD & B & $\mathrm{RL}, \mathrm{BL}$ \\
\hline & & 20 & 2.7 & 202 & 0.0116 & 25 & $\mathrm{M} / \mathrm{N} 1.2 \mathrm{~m}$ & 1k CCD & B & RL \\
\hline \multirow[t]{10}{*}{2009} & Jan & 06 & 1.6 & 143 & 0.0364 & 30 & Goe $0.5 \mathrm{~m}$ & SBIG ST-L & $\mathrm{B}$ & RL, US \\
\hline & & 22 & 3.7 & 334 & 0.0080 & 25 & $\mathrm{M} / \mathrm{N} 1.2 \mathrm{~m}$ & 1k CCD & B & RL \\
\hline & & 28 & 5.4 & 473 & 0.0147 & 20 & LOAO $1.0 \mathrm{~m}$ & $2 \mathrm{k} C \mathrm{CD}$ & B & SLK \\
\hline & & 30 & 6.6 & 662 & 0.0138 & 20 & LOAO $1.0 \mathrm{~m}$ & $2 \mathrm{k} C \mathrm{CD}$ & B & SLK \\
\hline & Feb & 18 & 2.4 & 202 & 0.0256 & 30 & Goe $0.5 \mathrm{~m}$ & SBIG ST-L & $\mathrm{B}$ & $\mathrm{RL}, \mathrm{TOH}$ \\
\hline & & 23 & 3.6 & 304 & 0.0126 & 25 & $\mathrm{M} / \mathrm{N} 1.2 \mathrm{~m}$ & 1k CCD & B & RL, US, MH \\
\hline & & 25 & 6.4 & 564 & 0.0076 & 25 & $\mathrm{M} / \mathrm{N} 1.2 \mathrm{~m}$ & $1 \mathrm{k} C \mathrm{CD}$ & B & RL \\
\hline & & 26 & 6.5 & 536 & 0.0111 & 25 & $\mathrm{M} / \mathrm{N} 1.2 \mathrm{~m}$ & $1 \mathrm{k} C \mathrm{CD}$ & B & RL, LN \\
\hline & & 27 & 6.6 & 556 & 0.0083 & 25 & $\mathrm{M} / \mathrm{N} 1.2 \mathrm{~m}$ & $1 \mathrm{k} C \mathrm{CD}$ & B & RL \\
\hline & & & 5.7 & 532 & 0.0067 & 15 & Asi $1.8 \mathrm{~m}$ & Afosc & no & $\mathrm{SB}, \mathrm{RC}$ \\
\hline
\end{tabular}


Table 3 (Continued)

\begin{tabular}{|c|c|c|c|c|c|c|c|c|c|c|}
\hline Year & Month & Day & $L[\mathrm{~h}]$ & $N$ & $\sigma_{\text {res }}$ & $\operatorname{Exp}[s]$ & Site $^{\mathrm{a}}$ & Inst. & Filter & Observer $^{b}$ \\
\hline \multirow[t]{27}{*}{2009} & \multirow[t]{16}{*}{ Mar } & 01 & 6.2 & 497 & 0.0090 & 25 & $\mathrm{M} / \mathrm{N} 1.2 \mathrm{~m}$ & $1 \mathrm{k} C \mathrm{CD}$ & B & RL \\
\hline & & 18 & 3.3 & 279 & 0.0060 & 25 & $\mathrm{M} / \mathrm{N} 1.2 \mathrm{~m}$ & $1 \mathrm{k} C \mathrm{CD}$ & B & RL \\
\hline & & 19 & 3.6 & 291 & 0.0098 & 25 & $\mathrm{M} / \mathrm{N} 1.2 \mathrm{~m}$ & $1 \mathrm{k} C C D$ & B & $\mathrm{RL}$ \\
\hline & & 20 & 2.4 & 243 & 0.0058 & 10 & $\mathrm{CA} 2.2 \mathrm{~m}$ & CAFOS & B & service (MA) \\
\hline & & 21 & 2.4 & 232 & 0.0068 & 10 & $\mathrm{CA} 2.2 \mathrm{~m}$ & CAFOS & B & service (MA) \\
\hline & & & 6.0 & 351 & 0.0150 & 30 & Asi $1.8 \mathrm{~m}$ & Afosc & B & $\mathrm{SB}, \mathrm{RC}$ \\
\hline & & 22 & 2.4 & 245 & 0.0049 & 10 & $\mathrm{CA} 2.2 \mathrm{~m}$ & CAFOS & B & service (MA) \\
\hline & & & 4.9 & 279 & 0.0140 & 30 & Asi $1.8 \mathrm{~m}$ & Afosc & B & $\mathrm{SB}, \mathrm{RC}$ \\
\hline & & 24 & 3.4 & 243 & 0.0092 & 25 & $\mathrm{M} / \mathrm{N} 1.2 \mathrm{~m}$ & $1 \mathrm{k} C \mathrm{CD}$ & B & RL \\
\hline & & 26 & 2.8 & 251 & 0.0081 & 25 & $\mathrm{M} / \mathrm{N} 1.2 \mathrm{~m}$ & $1 \mathrm{k} C \mathrm{CD}$ & B & RL, US \\
\hline & & & 3.9 & 396 & 0.0118 & 20 & LOAO $1.0 \mathrm{~m}$ & 2k CCD & B & SLK \\
\hline & & 27 & 1.3 & 160 & 0.0185 & 20 & LOAO $1.0 \mathrm{~m}$ & $2 \mathrm{k} C \mathrm{CD}$ & B & SLK \\
\hline & & 28 & 3.9 & 404 & 0.0172 & 20 & LOAO $1.0 \mathrm{~m}$ & $2 \mathrm{k} C \mathrm{CD}$ & B & SLK \\
\hline & & 29 & 2.9 & 309 & 0.0112 & 20 & LOAO $1.0 \mathrm{~m}$ & $2 \mathrm{k} C \mathrm{CD}$ & B & SLK \\
\hline & & 30 & 3.8 & 386 & 0.0144 & 20 & LOAO $1.0 \mathrm{~m}$ & $2 \mathrm{k} C \mathrm{CD}$ & B & SLK \\
\hline & & 31 & 4.0 & 399 & 0.0154 & 20 & LOAO $1.0 \mathrm{~m}$ & 2k CCD & B & SLK \\
\hline & \multirow[t]{2}{*}{ Sep } & 27 & 3.9 & 406 & 0.0132 & 25 & $\mathrm{M} / \mathrm{N} 1.2 \mathrm{~m}$ & $1 \mathrm{k} C \mathrm{CD}$ & B & RL \\
\hline & & 28 & 4.0 & 420 & 0.0117 & 25 & $\mathrm{M} / \mathrm{N} 1.2 \mathrm{~m}$ & $1 \mathrm{k} C \mathrm{CD}$ & B & RL \\
\hline & \multirow[t]{9}{*}{ Oct } & 16 & 4.0 & 412 & 0.0099 & 25 & $\mathrm{M} / \mathrm{N} 1.2 \mathrm{~m}$ & $1 \mathrm{k} C C D$ & B & $\mathrm{RL}$ \\
\hline & & 18 & 0.9 & 100 & 0.0109 & 25 & $\mathrm{M} / \mathrm{N} 1.2 \mathrm{~m}$ & $1 \mathrm{k} C \mathrm{CD}$ & B & RL \\
\hline & & 19 & 6.1 & 645 & 0.0119 & 25 & $\mathrm{M} / \mathrm{N} 1.2 \mathrm{~m}$ & $1 \mathrm{k} C \mathrm{CD}$ & B & RL \\
\hline & & 22 & 3.9 & 359 & 0.0076 & 10 & $\mathrm{CA} 2.2 \mathrm{~m}$ & CAFOS & B & service (AA) \\
\hline & & 23 & 3.6 & 334 & 0.0067 & 10 & $\mathrm{CA} 2.2 \mathrm{~m}$ & CAFOS & B & service (MA) \\
\hline & & 24 & 3.1 & 289 & 0.0061 & 10 & $\mathrm{CA} 2.2 \mathrm{~m}$ & CAFOS & B & service (MA) \\
\hline & & 25 & 2.8 & 297 & 0.0082 & 25 & $\mathrm{M} / \mathrm{N} 1.2 \mathrm{~m}$ & $1 \mathrm{k} C \mathrm{CD}$ & B & RL \\
\hline & & & 3.4 & 315 & 0.0078 & 10 & $\mathrm{CA} 2.2 \mathrm{~m}$ & CAFOS & B & service (MA) \\
\hline & & 26 & 3.0 & 279 & 0.0064 & 10 & $\mathrm{CA} 2.2 \mathrm{~m}$ & CAFOS & B & service (MA) \\
\hline
\end{tabular}

${ }^{a}$ Asi: Asiago $182 \mathrm{~cm}$ Copernico Telescope, CA: Calar Alto Observatory, Goe: Göttingen IAG $50 \mathrm{~cm}$ Telescope, Kon: Konkoly RCC Telescope, LOAO: Mt. Lemmon Optical Astronomy Observatory, Loi: Loiano $152 \mathrm{~cm}$ Telescope, M/N: MONET/North Telescope, MtB: Mt. Bigelow Kuiper Telescope, NOT: Nordic Optical Telescope, StB: Steward Observatory Bok Telescope, Tue: Tübingen $80 \mathrm{~cm}$ Telescope

${ }^{b}$ AA: Alberto Aguirre, ADB: Antonio De Blasi, AH: Agnes Hoffmann, BB: Benjamin Beeck, BL: Björn Loeptien, EMG: Elizabeth M. Green, LM: Luzma Montoya, LN: Lisa Nortmann, MA: Manuel Alises, MH: Markus Hundertmark, MP: Margit Paparó, PP: Péter Pápics, RC: Riccardo Claudi, RK: Renate Kruspe, RL: Ronny Lutz, RØ: Roy Østensen, SB: Serena Benatti, SD: Stefan Dreizler, SLK: Seung-Lee Kim, SS: Sonja Schuh, SW: Sascha Werhahn, TN: Thorsten Nagel, TOH: Tim-Oliver Husser, TS: Thorsten Stahn, US: Ulf Seemann, ZB: Zsófia Bognár

Table 4 The two main frequencies in HS 0444+0458 as obtained from the full 2008-2009 data set

\begin{tabular}{lccl}
\hline ID & Frequency $[\mu \mathrm{Hz}]$ & Period $[\mathrm{s}]$ & Amplitude $[\mathrm{mmi}]$ \\
\hline f1 & $7311.7522 \pm 0.0008$ & $136.76612 \pm 0.00016$ & $7.9 \pm 0.3$ \\
f2 & $5902.527 \pm 0.003$ & $169.4190 \pm 0.0008$ & $2.4 \pm 0.3$ \\
\hline
\end{tabular}

were obtained using a prescribed set of reference stars for each target. We then applied an extinction correction to the relative light curves using low-order polynomials. The timestamps of observations are given at mid-exposure, and they were barycentrically corrected as well as corrected
Table 5 The two main frequencies in HS 0702+6043 as obtained from the 2005 Calar Alto data set

\begin{tabular}{lllc}
\hline ID & Frequency $[\mu \mathrm{Hz}]$ & Period $[\mathrm{s}]$ & Amplitude $[\mathrm{mmi}]$ \\
\hline f1 & $2753.9618 \pm 0.0047$ & $363.1132 \pm 0.006$ & $27.2 \pm 0.2$ \\
f2 & $2606.0110 \pm 0.0241$ & $383.7282 \pm 0.0035$ & $5.3 \pm 0.2$ \\
\hline
\end{tabular}

for leap seconds. The BJD is stripped of the leading 24 and therefore appears as "Reduced" Julian Date in the figures (BRJD). The normalised relative intensities were given weights $1 / \sigma_{\text {res }}^{2}$ according to the local residual intensity scatter. The subsequent analysis of the weighted light curves was 


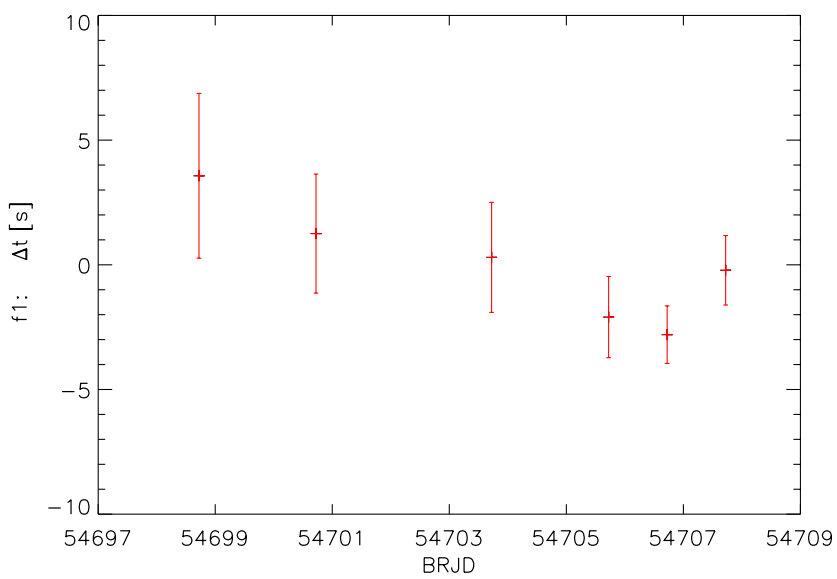

Fig. 1 TNG data on HS 0444+0458: O-C diagram for f1

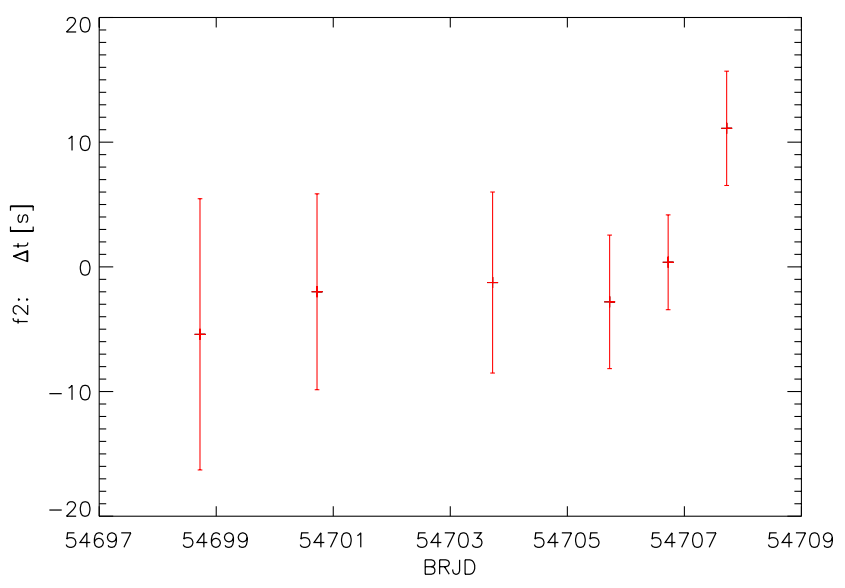

Fig. 2 TNG data on HS 0444+0458: O-C diagram for $\mathrm{f} 2$

done using Period0 4 by Lenz and Breger (2005). The errors for any frequencies, amplitudes, phases derived for various subsets of the data were calculated according to the prescription by Montgomery and O’Donoghue (1999).

\section{Coherence}

\subsection{Photometric monitoring of HS0444+0458}

We use the six-night data set from TNG in August 2008, which is the longest individual observing run and the one with the highest $\mathrm{S} / \mathrm{N}$ available for HS $0444+0458$, in order to examine the short-term stability of the two main pulsations in this star.

The frequencies used in this exercise were derived from the full data set, the values are as listed in Table 4. Keeping these frequencies fixed, we re-derived the best mean amplitude for the overall TNG data set, as well as the mean phase. Keeping both the frequencies and the amplitude fixed, we then turned to the individual nights to re-determine the

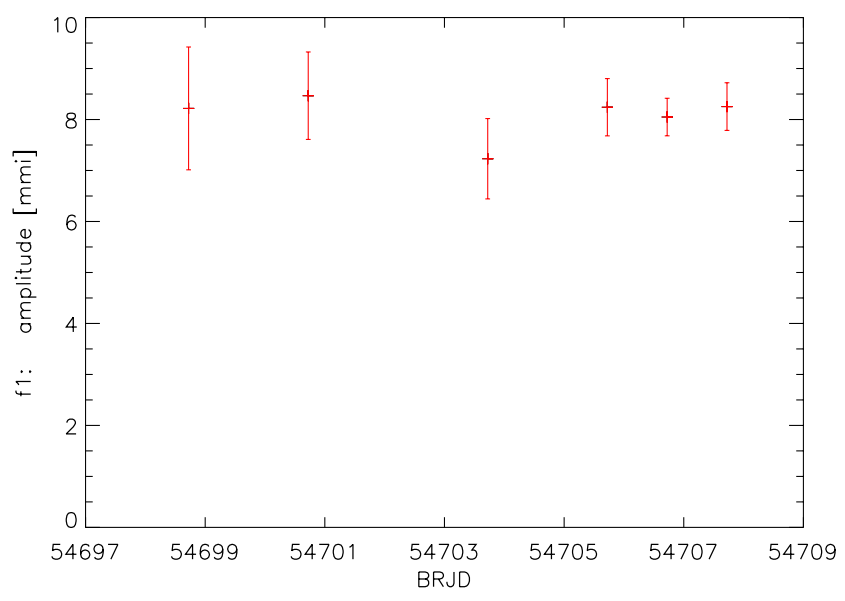

Fig. 3 TNG data on HS 0444+0458: amplitude variation for f1

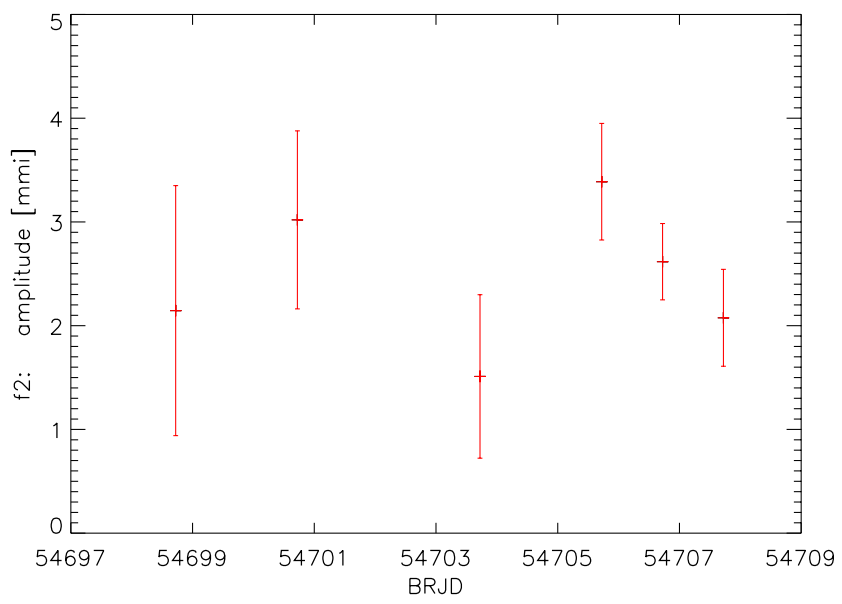

Fig. 4 TNG data on HS 0444+0458: amplitude variation for f2

phasings. The differences between the mean phase and the nightly phasings were converted into time lags, and the errors propagated from the error of the mean phase and the errors of the nightly phasings. The results for the frequencies $\mathrm{f} 1$ and $\mathrm{f} 2$ are shown in Figs. 1 and 2.

There are no serious discontinuities in the phases of the pulsations that would have to be attributed to instabilities in the pulsational behaviour of HS 0444+0458; all deviations are consistent with the uncertainties expected from the measurement uncertainties alone (short individual runs equal relatively large uncertainties in the phasing compared to phasings derived from an ensemble of several nights, as used for the construction of long-term $\mathrm{O}-\mathrm{C}$ diagrams).

The reasonably flat $\mathrm{O}-\mathrm{C}$ diagrams therefore show that HS 0444+0458's pulsations are coherent on this time scale. Extrapolating this result at the given overall frequency precision, the cycle count remains reliable during the $2-3$ month gaps in between the data sets obtained for HS $0444+0458$.

Similarly, Figs. 3 and 4 show the degree of variation in the amplitudes for $\mathrm{f} 1$ and $\mathrm{f} 2$ from night to night during the TNG run. 


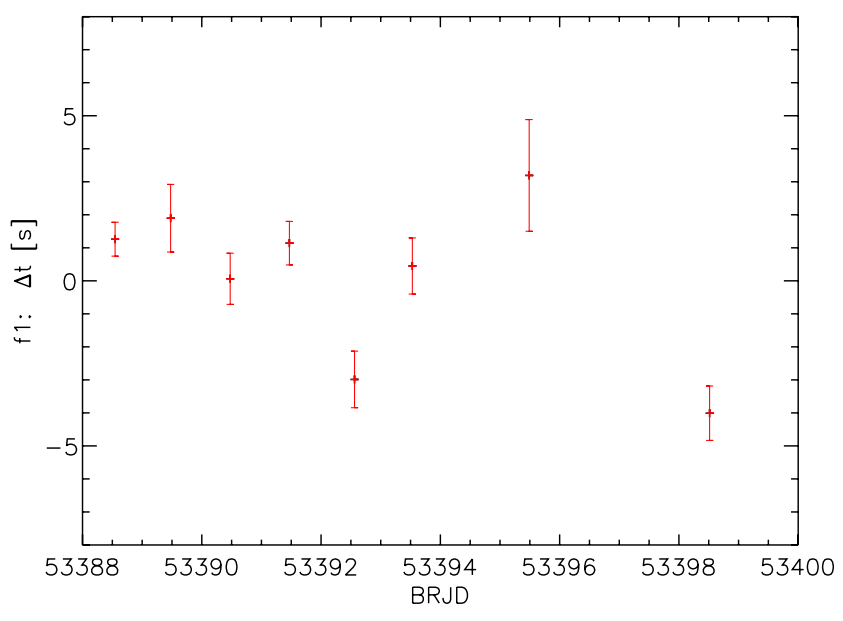

Fig. 5 Eight nights of Calar Alto data on HS 0702+6043: O-C diagram for $\mathrm{f} 1$

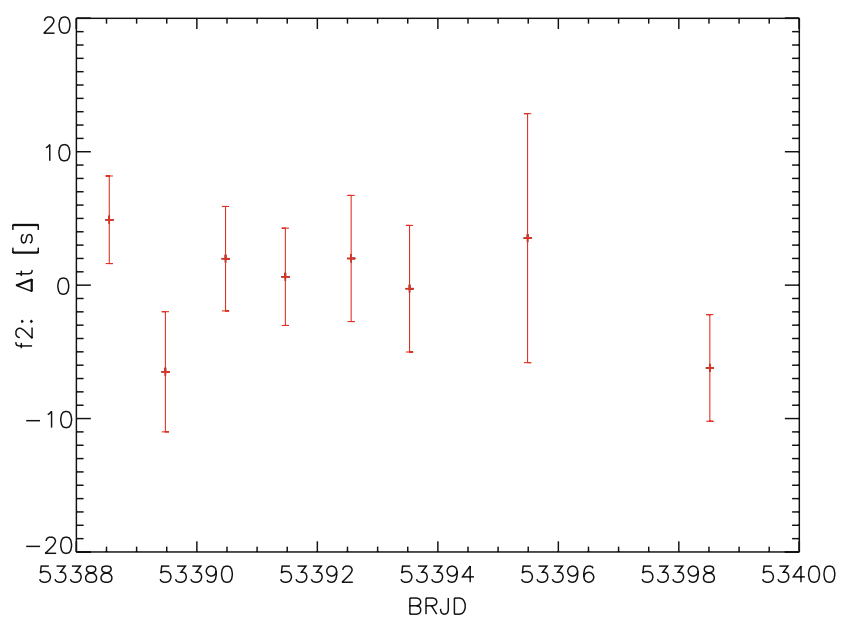

Fig. 6 Eight nights of Calar Alto data on HS 0702+6043: O-C diagram for $\mathrm{f} 2$

\subsection{Photometric monitoring of HS $0702+6043$}

The coherence and amplitude stability in HS $0702+6043$ were tested in two independent different data sets.

The first data set consists of eight nights of data obtained at Calar Alto in 2005. The two main rapid pulsations were recovered at the values given in Table 5 (compare also Lutz et al. 2008a). They were used in the same way as in the previous section to construct $\mathrm{O}-\mathrm{C}$ diagrams (Figs. 5 and 6) as well as to investigate the amplitude stability (Figs. 7 and 8) for $\mathrm{f} 1$ and $\mathrm{f} 2$ in HS 0702+6043.

Despite the longer data set and higher S/N (due to both a brighter target and higher pulsation amplitudes) and hence smaller error bars, the phases in HS 0702+6043 appear more scattered than in HS $0444+0458$, and the amplitude in particular for the main frequency $\mathrm{f} 1$ significantly less stable.

The second data set comprises the core 60 nights of the Mt. Bigelow run (see Francœur et al. 2010 for the frequency

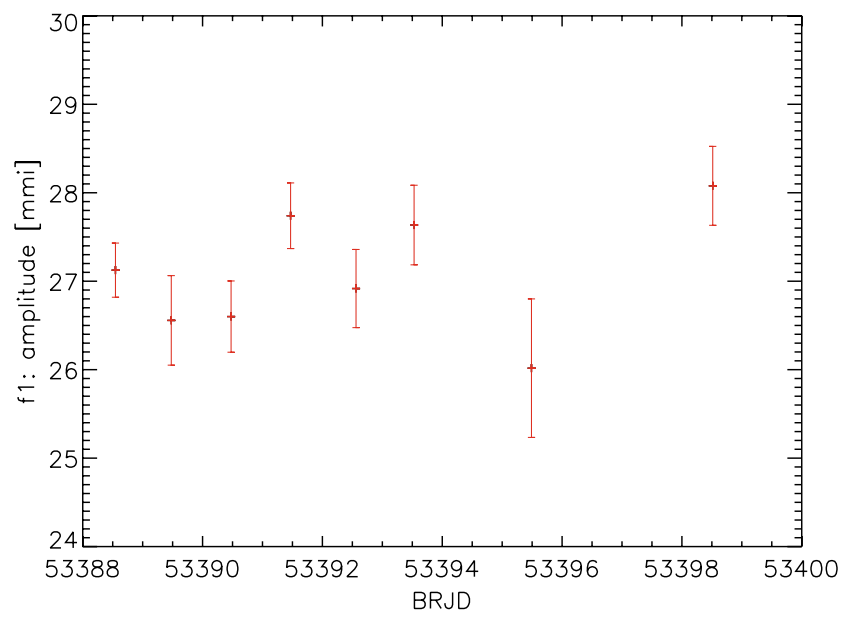

Fig. 7 Eight nights of Calar Alto data on HS 0702+6043: amplitude variation for $\mathrm{f} 1$

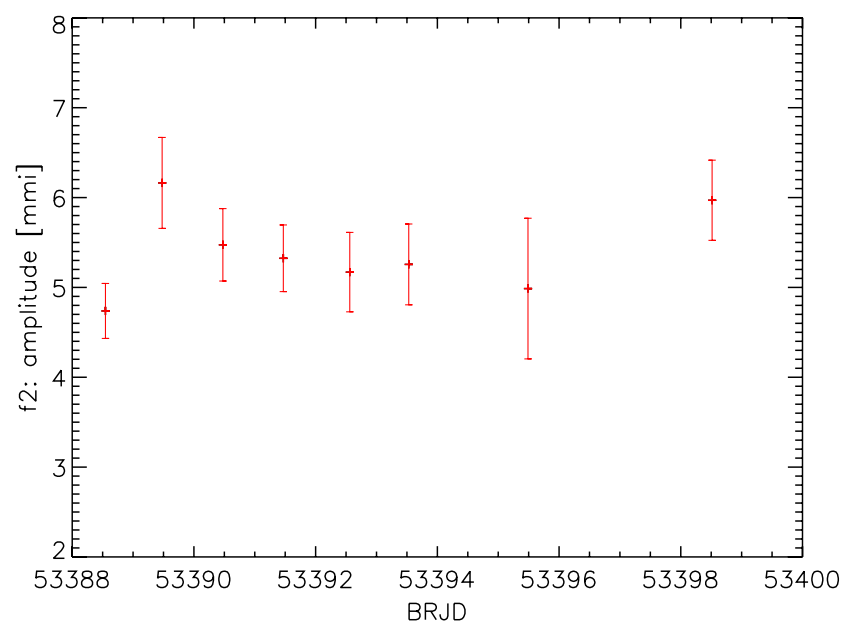

Fig. 8 Eight nights of Calar Alto data on HS 0702+6043: amplitude variation for $\mathrm{f} 2$

analysis). The most immediate and notable result from this data set is that HS $0702+6043$ shows a lot more frequencies both in the p- as well as in the g-mode domain than previously published.

We treated the data independently as above to obtain near-continuous $\mathrm{O}-\mathrm{C}$ diagrams spanning more than 100 days for $\mathrm{f} 1$ and $\mathrm{f} 2$ (Figs. 9 and 10) as well as diagrams of the evolution of the corresponding pulsation amplitudes (Figs. 11 and 12) during this time. The results for the amplitude variation confirm those obtained by Francœur et al. (2010).

All of these figures reveal a rather complex behaviour that needs to be discussed in detail. The $\mathrm{O}-\mathrm{C}$ diagram for f1 shows a significant trend which looks more or less linear (which is also seen in the associated amplitude). Normally this would be a telltale sign for a mean frequency chosen at a slightly wrong value, which can be corrected to subsequently get rid of the trend. However, all attempts 


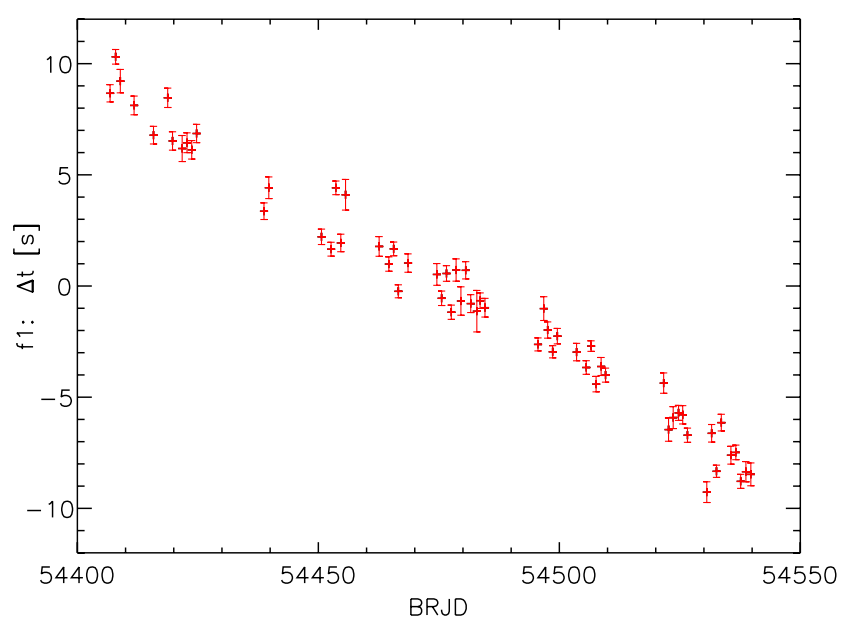

Fig. 960 nights of Mt. Bigelow data on HS 0702+6043: O-C diagram for $\mathrm{f} 1$

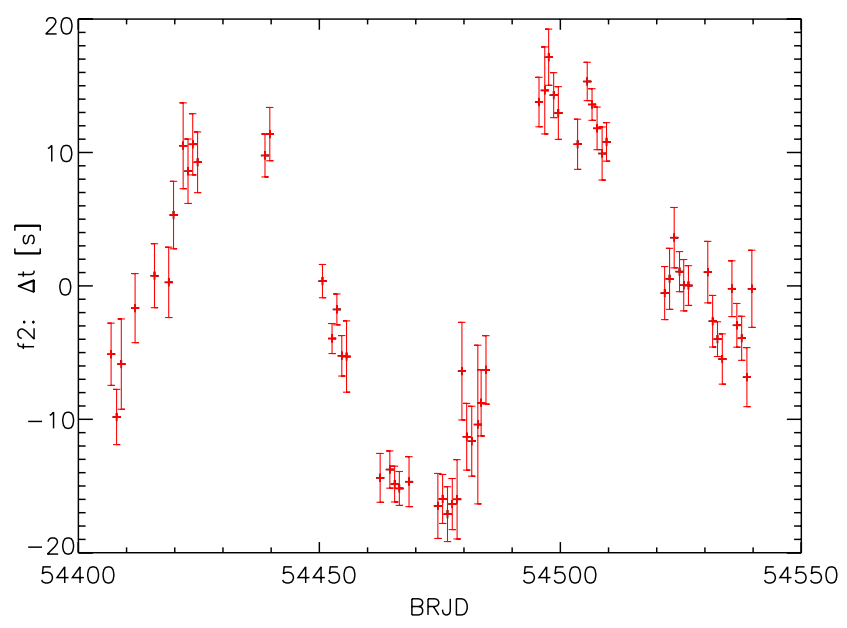

Fig. 1060 nights of Mt. Bigelow data on HS 0702+6043: O-C diagram for $\mathrm{f} 2$

to "improve" the frequency have so far proven unsuccessful to eliminate this trend (a result consistent with the mean solution for the full 2004-2009 data), so it must represent a higher-order effect.

The $\mathrm{O}-\mathrm{C}$ diagram for $\mathrm{f} 2$ shows an even more complex behaviour, with roughly oscillatory shape (with a very similar evolution in the amplitude), indicative of beating. With less than two full "beating cycles" covered even by this very extensive data set, however, the hypothetical multiplet structure potentially generating this beating still cannot be adequately resolved.

It is worth noting that the $\mathrm{O}-\mathrm{C}$ diagrams and amplitude variation results do not change when increasingly more frequencies are included simultaneously in the analysis (up to 18 frequencies tested); this does not include, however, hypothetical additional very close frequencies within the frequency resolution of frequencies already considered.

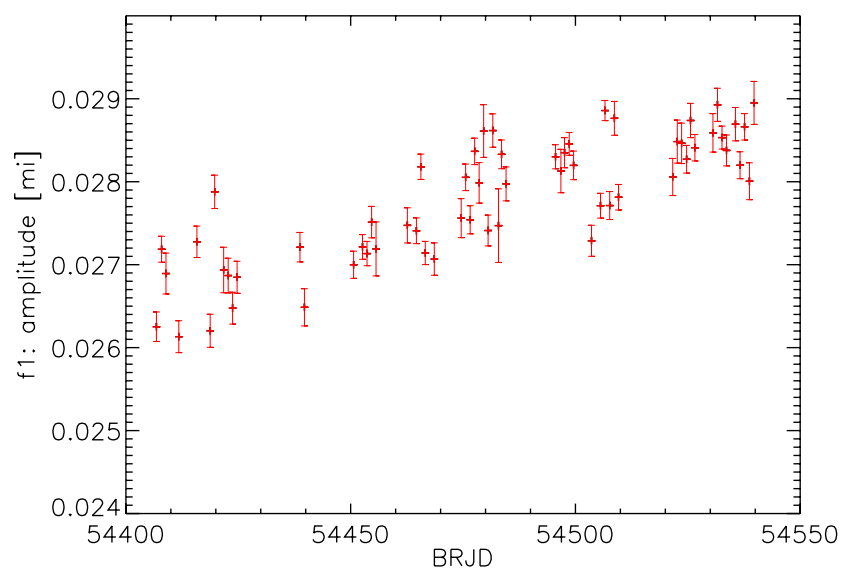

Fig. 1160 nights of Mt. Bigelow data on HS 0702+6043: amplitude variation for $\mathrm{f} 1$

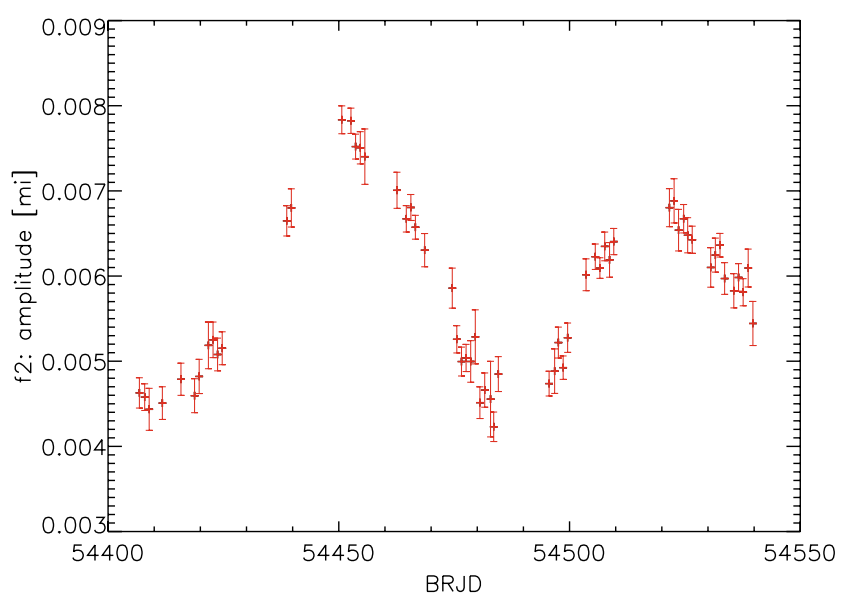

Fig. 1260 nights of Mt. Bigelow data on HS 0702+6043: amplitude variation for $\mathrm{f} 2$

It would therefore be tempting to claim that this data set reveals first indications for what the EXOTIME program is, after all, looking for: drifts or even periodic residuals in the $\mathrm{O}-\mathrm{C}$ diagrams that can only be explained by the influence of substellar companions. However, at most one of the trends in the $\mathrm{O}-\mathrm{C}$ diagrams could be attributed to one or more unseen companion, as the light travel time would have to change in exactly the same way and with exactly the same amplitude for all pulsations, independently of their other properties. Such a similarity in the patterns of the $\mathrm{O}-\mathrm{C}$ diagrams for different pulsations has however not been uncovered in this analysis so far, and all remaining irregularities would still have to be explained differently. In the meantime, it remains unclear if external effects (including unresolved close frequencies) causes these features or if the pulsations truly drift very slowly in amplitude, phase or period.

Finally, the fact that the $\mathrm{O}-\mathrm{C}$ diagrams for the much shorter Calar Alto data alone does not look so bad (although not as good as that for HS 0444+0458) by itself, while the $\mathrm{O}-\mathrm{C}$ diagrams for the much longer continuous Mt. Bigelow 
data set proves the star's pulsations to be much more complicated, reminds us that all conclusions derived from shorter runs should be interpreted with a fair amount of caution.

\subsection{Follow-up observations of HS 2201+2610}

The $\mathrm{O}-\mathrm{C}$ diagrams, as well as pulsational amplitudes, derived for HS 2201+2610 have been published elsewhere. An extension of the diagrams for $\mathrm{f} 1$ and $\mathrm{f} 2$ of this star is work in progress, with significant amounts of new data available by now. In addition to the continuing long-term photometric monitoring data in one filter, multi-colour data and time-resolved spectra have been obtained. Schuh et al. (2009) sketch an idea of how to combine such observations to get an estimate of the orbital inclination $i$ for the companion, which would allow to transform the current $m_{2} \sin i$ measurement into a true mass $m_{2}$. We note that efforts in this direction have, however, only indicated so far that (a) HS $2201+2610$ is probably a slow rotator and that it (b) has very small pulsational radial velocity amplitudes.

\section{Long-term O-C diagrams}

While the long-term O-C diagrams for HS $2201+2610$ are available elsewhere, their construction for HS $0702+6043$ is work in progress, and is currently still hampered by the difficulties detailed in Sect. 3.2.

We therefore focus on presenting the preliminary $\mathrm{O}-\mathrm{C}$ diagrams for HS $0444+0458$ here. The overall coverage (a total of 8 month of data) is much shorter here in comparison to HS $2201+2610$ and HS $0702+6043$, so that obviously no results for $\dot{P}$ or even more complex residuals can be presented yet.

The very important preliminary result for this star, however, is that the $\mathrm{O}-\mathrm{C}$ diagrams for both its frequencies $\mathrm{f} 1$ and f2 look fairly flat within the error bars over the full 8 month period, as can be seen in Figs. 13 and 14. This means that HS 0444+0458 is very well suited as a EXOTIME target and definitely worth further follow-up.

\section{Summary}

The main result for HS $0444+0458$ so far is that the O-C diagram for its main pulsation frequency f1 is very wellbehaved. The $\mathrm{O}-\mathrm{C}$ diagram for $\mathrm{f} 2$ is also approximately flat, but with much larger errors and hence less significance due to the lower $\mathrm{S} / \mathrm{N}$. We conclude that the degree of coherence of the pulsations in HS 0444+0458 confirms it as a good target for long-term monitoring. The next few years will show if this reveals a measurable $\dot{P}$ for the star and/or the signature of an orbiting (planetary) companion.

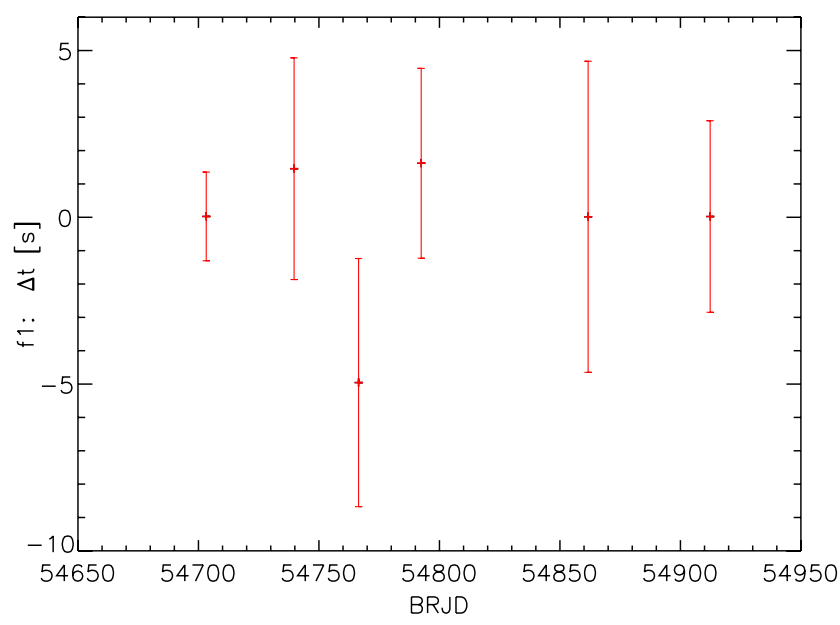

Fig. 13 All data on HS 0444+0458: O-C diagram for f1

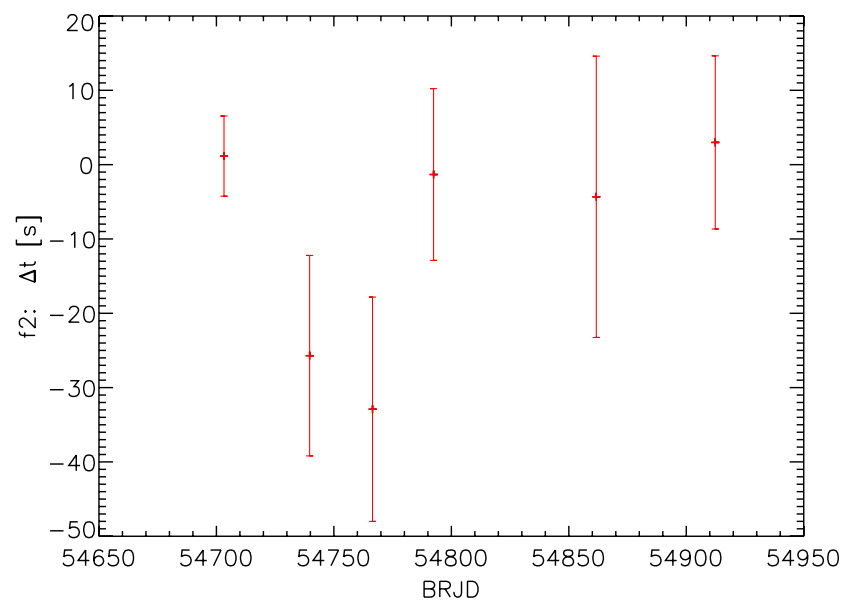

Fig. 14 All data on HS 0444+0458: O-C diagram for f2

For HS $0702+6043$, previously thought to show a relatively simple spectrum in the p-mode domain, it turns out (Francœur et al. 2010, and the analysis in this work) that a significant amount of work must first be put into developing a very good understanding of the pulsational behaviour before these pulsations can be used as a tool to derive $\dot{P}$ or even search for companions.

\section{Discussion}

The increasing number of detections of low-mass companions (Silvotti et al. 2007; Lee et al. 2009; Qian et al. 2009; Geier et al. 2009; Geier 2010) may indicate the existence of a previously undiscovered population of substellar and planetary bodies around subdwarf B stars. This has renewed interest in the question, initially raised by Soker (1998), whether planets might be relevant for the formation of subdwarf B stars. 
To discuss the possible influence of a substellar companion on the primary star's evolution on the red giant branch, the above systems must be considered individually. In the case of HS 2201+2610 (Silvotti et al. 2007), for instance, the detected planet is too far out to have strongly influenced the envelope ejection process. On the other hand, when a star forms planets, it often forms full planetary systems with several members, so that a remaining detectable far-out planet may serve as a tracer of previously present closer-in planets, possibly destroyed during the RGB phase. In this sense, the detection of V391 Pegasi b could be interpreted as backing up the suggestion that planets can indeed enhance the mass loss on the RGB in such a way that the subsequent formation of a subdwarf B star is favoured (Soker 2010). The detection of HD 149382 b (Geier et al. 2009; Geier 2010) may constitute more direct evidence, with the actual object having undergone the common envelope phase still present today.

The situation is different for the tertiary bodies in the eclipsing close binary systems HW Vir and HS 0705+6700 (Lee et al. 2009; Qian et al. 2009). The "problem" of "missing" companions that would be needed to explain the formation of the primary subdwarf B star through one of the accepted binary evolution channels has never existed here, as suitable secondary bodies were already known.

The detections of low-mass objects around apparently single sdB stars are therefore the more relevant ones when it comes to the discussion whether such companions might be efficient in forming subdwarf B stars. In particular, the amount of angular moment transferred and hence the modification of the ejection efficiency during a common envelope phase are a matter of debate among theorists. In alternative scenarios, the existence of planets around subdwarf B stars is explained by formation processes leading to second generation planets.

The EXOTIME program has the potential to add to the empirical data available on which such a debate must ultimately be based.

\footnotetext{
Acknowledgements The authors thankfully acknowledge the contributions by J. Dittmann, A.G. Fay, J. Laird, C.M. Limbach, J. Portouw, M. Revelle, J.M. Sierchio, S.M. Story, C. Stratton, A. Strom, P. Wroblewski (all Steward Observatory), who helped obtain the Mt. Bigelow data set. S.S. acknowledges funding through the EberhardKarls-Universität Tübingen's Teaching Equality program, and also thanks the DAAD for allocating a travel grant to attend the Forth Meeting on Hot Subdwarfs and Related Objects where this paper was presented. The work of R.L. on this project is funded by a stipend from the IMPRS PhD program at MPS Katlenburg-Lindau. C.R.L. acknowledges an Ángeles Alvariño contract of the regional government Xunta de Galicia. Amongst others, the work in this paper is

- based on observations collected at the Centro Astronómico Hispano Alemán (CAHA) at Calar Alto, operated jointly by the Max-Planck Institut für Astronomie and the Instituto de Astrofísica de Andalucía (CSIC)
}

- based on observations made with the Italian Telescopio Nazionale Galileo (TNG) operated on the island of La Palma by the Fundación Galileo Galilei of the INAF (Istituto Nazionale di Astrofisica) at the Spanish Observatorio del Roque de los Muchachos of the Instituto de Astrofisica de Canarias

- based on data obtained with the MOnitoring NEtwork of Telescopes (MONET), funded by the 'Astronomie \& Internet' program of the Alfried Krupp von Bohlen und Halbach Foundation, Essen, and operated by the Georg-August-Universität Göttingen, the McDonald Observatory of the University of Texas at Austin, and the South African Astronomical Observatory

Note This paper contains the content of three contributions originally presented as

- Status report on the EXOTIME program

- EXOTIME photometric monitoring of HS 0444+0458

- EXOTIME photometric monitoring of HS 0702+6043

Open Access This article is distributed under the terms of the Creative Commons Attribution Noncommercial License which permits any noncommercial use, distribution, and reproduction in any medium, provided the original author(s) and source are credited.

\section{References}

Christensen-Dalsgaard, J., Kjeldsen, H., Mattei, J.A.: Astrophys. J. Lett. 562, 141 (2001). doi:10.1086/338194

Costa, J.E.S., Kepler, S.O.: Astron. Astrophys. 489, 1225 (2008). doi:10.1051/0004-6361:20079118

Costa, J.E.S., Kepler, S.O., Winget, D.E.: Astrophys. J. 522, 973 (1999). doi:10.1086/307655

Dreizler, S., Schuh, S.L., Deetjen, J.L., Edelmann, H., Heber, U.: Astron. Astrophys. 386, 249 (2002). doi:10.1051/0004-6361: 20020200

Francœur, M., Fontaine, G., Green, E.M., et al.: Astrophys. Space Sci. (2010, this issue)

Geier, S.: Astrophys. Space Sci. (2010, this issue)

Geier, S., Edelmann, H., Heber, U., Morales-Rueda, L.: Astrophys. J. Lett. 702, 96 (2009). doi:10.1088/0004-637X/702/1/L96

Kepler, S.O., Winget, D.E., Nather, R.E., Bradley, P.A., Grauer, A.D., Fontaine, G., Bergeron, P., Vauclair, G., Claver, C.F., Marar, T.M.K., Seetha, S., Ashoka, B.N., Mazeh, T., Leibowitz, E., Dolez, N., Chevreton, M., Barstow, M.A., Clemens, J.C., Kleinman, S.J., Sansom, A.E., Tweedy, R.W., Kanaan, A., Hine, B.P., Provencal, J.L., Wesemael, F., Wood, M.A., Brassard, P., Solheim, J., Emanuelsen, P.: Astrophys. J. Lett. 378, 45 (1991). doi:10.1086/186138

Kepler, S.O., Mukadam, A., Winget, D.E., Nather, R.E., Metcalfe, T.S., Reed, M.D., Kawaler, S.D., Bradley, P.A.: Astrophys. J. Lett. 534, 185 (2000). doi:10.1086/312664

Kepler, S.O., Costa, J.E.S., Castanheira, B.G., Winget, D.E., Mullally, F., Nather, R.E., Kilic, M., von Hippel, T., Mukadam, A.S., Sullivan, D.J.: Astrophys. J. 634, 1311 (2005). doi:10.1086/497002

Kilkenny, S.: Astrophys. Space Sci. (2010, this issue)

Lee, J.W., Kim, S.L., Kim, C.H., Koch, R.H., Lee, C.U., Kim, H.I., Park, J.H.: Astron. J. 137, 3181 (2009). doi:10.1088/0004-6256/ $137 / 2 / 3181$

Lenz, P., Breger, M.: Commun. Asteroseismol. 146, 53 (2005). doi: $10.1553 /$ cia146s 53

Lutz, R., Schuh, S., Silvotti, R., Dreizler, S., Green, E.M., Fontaine, G., Stahn, T., Hügelmeyer, S.D., Husser, T.: In: Heber, U., Jeffery, C.S., Napiwotzki, R. (eds.) Hot Subdwarf Stars and Related Objects. Astronomical Society of the Pacific Conference Series, vol. 392, p. 339. Astronomical Society of the Pacific, San Francisko (2008a) 
Lutz, R., Schuh, S., Silvotti, R., Kruspe, R., Dreizler, S.: Commun. Asteroseismol. 157, 185 (2008b)

Lutz, R., Schuh, S., Silvotti, R., Kruspe, R., Dreizler, S.: Commun. Asteroseismol. 159, 94 (2009a)

Lutz, R., Schuh, S., Silvotti, R., Bernabei, S., Dreizler, S., Stahn, T., Hügelmeyer, S.D.: Astron. Astrophys. 496, 469 (2009b). doi:10.1051/0004-6361/20079257

Montgomery, M.H., O’Donoghue, D.: Delta Scuti Star Newsl. 13, 28 (1999)

Mullally, F., Winget, D.E., De Gennaro, S., Jeffery, E., Thompson, S.E., Chandler, D., Kepler, S.O.: Astrophys. J. 676, 573 (2008). doi:10.1086/528672

Østensen, R., Solheim, J.E., Heber, U., Silvotti, R., Dreizler, S., Edelmann, H.: Astron. Astrophys. 368, 175 (2001a). doi:10.1051/ 0004-6361:20000488

Østensen, R., Heber, U., Silvotti, R., Solheim, J.E., Dreizler, S., Edelmann, H.: Astron. Astrophys. 378, 466 (2001b). doi:10.1051/ 0004-6361:20011218

Qian, S.B., Zhu, L.Y., Zola, S., Liao, W.P., Liu, L., Li, L.J., Winiarski, M., Kuligowska, E., Kreiner, J.M.: Astrophys. J. Lett. 695, 163 (2009). doi:10.1088/0004-637X/695/2/L163
Reed, M.D., Terndrup, D.M., Zhou, A.Y., Unterborn, C.T., An, D., Eggen, J.R.: Mon. Not. R. Astron. Soc. 378, 1049 (2007). doi:10.1111/j.1365-2966.2007.11839.x

Schuh, S., Huber, J., Dreizler, S., Heber, U., O'Toole, S.J., Green, E.M., Fontaine, G.: Astron. Astrophys. 445, 31 (2006). doi:10.1051/0004-6361:200500210

Schuh, S., Kruspe, R., Lutz, R., Silvotti, R.: Commun. Asteroseismol. 159, 91 (2009)

Silvotti, R., Janulis, R., Schuh, S.L., Charpinet, S., Oswalt, T., Silvestri, N., Gonzalez Perez, J.M., Kalytis, R., Meištas, E., Ališauskas, D., Marinoni, S., Jiang, X.J., Reed, M.D., Riddle, R.L., Bernabei, S., Heber, U., Bärnbantner, O., Cordes, O., Dreizler, S., Goehler, E., Østensen, R., Bochanski, J., Carlson, G.: Astron. Astrophys. 389, 180 (2002). doi:10.1051/0004-6361:20020584

Silvotti, R., Schuh, S., Janulis, R., Solheim, J.E., Bernabei, S., Østensen, R., Oswalt, T.D., Bruni, I., Gualandi, R., Bonanno, A., Vauclair, G., Reed, M., Chen, C.W., Leibowitz, E., Paparo, M., Baran, A., Charpinet, S., Dolez, N., Kawaler, S., Kurtz, D., Moskalik, P., Riddle, R., Zola, S.: Nature 449, 189 (2007). doi: 10.1038/nature06143

Soker, N.: Astron. J. 116, 1308 (1998). doi:10.1086/300503

Soker, N.: Astrophys. Space Sci. (2010, this issue) 